\title{
Multitasking Bacterial Moonlighting Proteins in Bacterial Virulence and Infectious Disease
}

\author{
Aus Molan \\ Department of Research and in-vitro Diagnostics/Abacus ALS/New Zealand
}

\begin{abstract}
Men may not be able to multitask, but it seems that proteins can. Although the one gene-one protein-one function hypothesis is well ingrained in the Central Dogma, it has become clear during the past decade that many proteins have one or more unique functions in addition to their primary biological action. These proteins perform multiple independent and often unrelated roles without partitioning these functions into different domains of the protein. This ability of proteins to exhibit more than one function is referred to as protein moonlighting, in analogy to moonlighting people who have multiple jobs. Many well known proteins are now known to moonlight, for example, metabolic enzymes, which in addition to their catalytic function are involved in fully unrelated processes such as autophagy, protein transport or DNA maintenance. Additionally, a growing number of bacterial species are also being found to express moonlighting proteins and the moonlighting activities of such proteins can contribute to bacterial virulence behaviour. This review recapitulates the current knowledge of moonlighting proteins and highlights the multiple roles displayed by a range of bacterial proteins. Also discussed is the role moonlighting activity plays in the virulence characteristics of a number of important human pathogens, including Streptococcus pyogenes, Streptococcus pneumoniae, Helicobacter pylori, and Mycobacterium tuberculosis. The medical relevance and noteworthy implications for fundamental biomedical research are also reviewed. It appears that only the tip of the bacterial moonlighting iceberg has been seen and in the near future we will see numerous new discoveries of bacterial moonlighting proteins with roles in bacterial virulence. Moonlighting is a phenomenon that illustrates nature's ingenuity. It is a source of stimulation that should remind scientists to always keep the unexpected in mind, even on well-known ground.
\end{abstract}

Keywords: Molecular evolution, moonlighting proteins, multitasking proteins, bacterial virulence; infectious disease, biomedical research.

\section{Introduction}

The ground-breaking work of Garrod [1] and Tatum and Beadle [2], which was carried out some time before the identification of the structure of DNA, led to the general expectation that every gene encodes a single protein, and that each protein serves a single function. However, in recent years, this logical view of biology has turned out to be overly simplistic. When the human genome sequence became available, it was found that encodes far less proteins than expected for such a complex organism with many different cell types [3]. The human genome encodes approximately 25,000 proteins and up until the 1980 s, no evidence for the alternative hypothesis was proposed- that individual proteins can have more than one biological activity.

However, in the late 1980's, two scientists described an avian eye lens crystallin protein in the duck that turned out to be a previously known metabolic enzyme, argininosuccinate lyase [5]. Piatigorsky and Wistow [5] proposed the term 'gene sharing' to describe this phenomenon, in which one gene product has more than one function. Consequent work showed that the lens crystallins in several mammals, birds, geckos, and other species were found to be the same proteins as several cytosolic enzymes [5, 6]. Furthermore, Piatigorsky [5] also realised that this gene sharing had consequences for the transcriptional control of the shared genes. Although considered the pioneer in the field of protein molecular biology, Piatigorsky's gene sharing hypothesis failed to attract the attention it deserved and the term gene sharing is seldom used in current literature [4].

While earlier descriptions of protein moonlighting have been reported, this phenomenon was not explicitly recognised [4]. Glyceraldehyde-3-phosphate dehydrogenase (GAPD), for example, is one of the more accomplished moonlighting proteins, presently well recognised for having functions in the nucleus [7]. Reports that GAPD could bind single, but not double, stranded DNA were published as early as 1980 [8]. The term protein moonlighting can be traced back Campbell and Scanes who reported that somatostatin and growth hormone, had immune-modulatory activity as well as their typical neuropeptide function and termed this activity 'moonlighting' [4]. Successive use of the expression 'moonlighting' was in an editorial describing a paper that reported the involvement of yeast enzymes in repairing DNA double strand breaks also involved in the maintenance of telomeres [9]. Of note however, is the work of Constance Jeffery who has publicised the concept of protein moonlighting and endeavoured to convey some description to this novel area of protein biology [10]. 
The phrase moonlighting is defined as having a second job, usually at night, in addition to one's regular daytime occupation. Jeffery attempted to categorise the various functional facets of moonlighting proteins and limit their definition in 1999 [10]. By description, moonlighting proteins are very particular multifunctional proteins. The key characteristic of these proteins is the expression of clearly distinct biological activities. This may be an attribute of the original active site of the protein or may be due to the evolution of other sites on the protein with biological function [4]. Moonlighting proteins execute multiple independent, often unrelated, functions without partitioning these functions into different protein domains (in other words, two or more functions performed by a single polypeptide chain) [11]. Accordingly, proteins generated by homologous but non-identical proteins, gene fusions, splice variants, protein fragments, protein decoration variants, and proteins operating in different locations or utilising different substrates are not considered moonlighting proteins $[8,11]$. An additional chief standard for moonlighting proteins is the independency of both functions. This means that in-activation of one of the functions (e.g. by mutation) should not affect the second function and vice versa [11]. Also, moonlighting should not be confused with pleiotropism. Hubert and van der Klei define pleiotropic effects as the result of single function inactivation, which participates in multiple cellular processes, e.g. a protein that has several interaction partners in different pathways or an enzyme which is important in several metabolic pathways [11]. Instead, moonlighting proteins execute multiple functions, which differ mechanistically. These alternative functions are mostly related to cellular localisation, cell type, oligomeric state, and cellular concentration of ligands, substrates, cofactors, products or post-translational modifications [3, 4, 8, 12-18]. Enzymes which utilise two different substrates or have two metabolic functions are categorised as bi-functional enzymes [19]. Copley has coined the term 'catalytic promiscuity' to describe the situation of an enzyme which has an active site that is able to catalyse two different reactions [18]. These forms of moonlighting will not be discussed in this review.

This report reviews the current literature on moonlighting proteins and highlights the multiple roles displayed by a range of bacterial proteins. Emphasis will be placed on the role that such moonlighting activity plays in the virulence characteristics of a number of important human pathogens, including Streptococcus pyogenes, Streptococcus pneumoniae, Helicobacter pylori, and Mycobacterium tuberculosis. Additionally, the medical relevance and noteworthy implications for fundamental biomedical research and human health are examined. The existence of moonlighting functions is a major challenge in protein biology. Even well studied proteins might harbour additional functions that have yet to be discovered and the discovery of many moonlighting functions has so far been completely unanticipated. This report skims the surface of this field and it is likely that in the near future we will see numerous new discoveries of bacterial moonlighting proteins with roles in bacterial virulence.

\section{Moonlighting Proteins In Bacterial Virulence And Disease}

Seventeen years after the introduction of the protein moonlighting hypothesis, it is evident that the bacterium is one of the major beneficiaries of this evolutionary mechanism. A significant number of examples of bacterial moonlighting proteins have been reported with many of these proteins playing a role in the virulence properties of bacteria. Thus, it is important to recognise this phenomenon in bacteriology. However, the fact that not all protein homologues moonlight [15], or they may have different moonlighting functions, complicates this understanding of protein moonlighting. One must appreciate this fact when reading the moonlighting literature. Major groups of moonlighting proteins that are involved in bacterial virulence include: (i) metabolic enzymes of the glycolytic pathway; and (ii) molecular chaperones and protein-folding catalysts.

\section{II.1 Moonlighting bacterial glycolytic enzymes}

The glycolytic pathway exists in the three kingdoms of life, and is believed to have developed early in the evolution of life. A large number of glycolytic pathway enzymes have been found to act as moonlighting proteins. The Gram-positive bacteria streptococci and staphylococci have most of the enzymes of the glycolytic pathway on their cell surfaces [20]. Such reports have been criticised as being the result of the binding of enzymes to the bacterial surface as an outcome of bacterial death and the release of such enzymes. The reason for this is that there is no currently known mechanism for the selective release of glycolytic enzymes. However, Boel et al. [21] suggest that there is strong evidence that the cell surface enzyme glyceraldehyde-3-phosphate dehydrogenase (GAPDH) of S.pyogenes is there as part of an undescribed system for secreting cytoplasmic enzymes. This hypothesis is supported by the studies of the surface location of GAPDH in Lactobacillus crispatus, which reveals that soluble GAPDH does not bind to the surface of the intact organism [22].

The glycolytic enzyme GAPDH, found on the cell surface of S.pyogenes, was the first moonlighting bacterial protein to be described in studies that were conducted before the moonlighting concept was introduced [23]. It was shown that GAPDH is tightly attached to surfaces of cells by a mechanism which is yet not defined [4]. From S.pyogenes, the isolated and purified GAPDH was established to have a binding capacity to an array of ligands including the major host adhesive glycoprotein, lysozyme, and fibronectin, as well as the cytoskeletal 
proteins, actin and myosin [23]. Hence, GAPDH is truly a prototypic bacterial moonlighting protein as many bacterial moonlighting proteins are cytoplasmic proteins found on the cell surface capable of adhesive functionality. Jeffery [24] has proposed one way of identifying moonlighting proteins by proteomic analysis of proteins in unusual sites. However, the identification of proteins on the cell surface, for example, is not an indication that it is automatically a moonlighting protein. Nevertheless, it is a suitable place to start for such an analysis. Interestingly, using use of proteomic analysis over the last decade has shown that a number of bacteria have cytosolic proteins that exist on the outer surface of the bacteria. Various different streptococci have been analysed in this manner and there seems to be an interesting variation and similarity in the populations of secreted cell surface proteins. For example, in Streptococcus agalactiae, the following cytosolic proteins were identified on the outer surface of the cell: glucose-6-phosphate isomerase, enolase, non-phosphorylating GAPDH, purine nucleotide phosphorylase, ornithine carbamoyltransferase, cysteine synthase, superoxide dismutase, chaperonin 60, and DnaK [20]. In Strepococcus oralis, 27 cell surface proteins were identified including the glycolytic enzymes: fructose bisphosphate aldolase, triosephosphate isomerase, GAPDH, enolase and phosphoglycerate kinase [4]. Additionally, Strepococcus pneumoniae also has a number of glycolytic enzymes on the bacterial cell surface including lactate dehydrogenase. Of particular note is that antibodies to several of the above mentioned pneumococcal proteins are also found in healthy persons [25].

The prototypic moonlighting protein Enolase, found in both prokaryotes and eukaryotes, has been shown to have roles in a variety of human diseases [26]. A growing range of microbes have been reported to have cell surface enolases which have moonlighting functions with human cells, such as neurons and endothelial cells, and also yeasts and protozoa having cell surface enolases [26]. The cell surface enolase of group A streptococci have had the most attention due to the work of Pancholi and Fischetti [23] who can be credited for much of the understanding of streptococcal cell surface enolase and its role in the virulence of this organism. It has been shown that enolase is present on the surface of most streptococci [27] including S. pneumoniae [28]. Plasminogen binding to the surface of pneumococci enables these bacteria to penetrate a synthetic basement membrane gel which is believed to be important for the invasion of this organism that results in meningitis [28]. Mutation at the C-terminal residues or these residues plus the internal site (residues 248 to 256) of S. pneumoniae enolase results in the production of a protein missing the internal plasminogen binding site which significantly reduces the virulence of the organism in a mouse nasal inoculation model [28]. Additionally, when the enolase of the Aeromonas hydrophila, which also binds to plasminogen, is used to immunise mice, the pathology consequent upon infection with this bacterium is noticeably decreased [29].

Therefore, we can surmise that cell surface enolases contribute to bacterial virulence. From this perspective, it is interesting that Dinis and colleagues [30] have reported that recombinant enolase from Streptococcus obrinus, found in oral cavities, is an immunosuppressive agent which if administered orally, can be used to protect against dental caries in rats [30]. Moreover, the cell surface enolase of Lactobacillus jensenii, a vaginal Gram-positive bacillus commensal organism, is a powerful inhibitor of the adherence of Neisseria gonorrhoeae to epithelial cells [31].

\section{II.2 Bacterial molecular chaperons modulate mononuclear phagocyte function}

The ability of bacterial molecular chaperones to activate or inhibit mononuclear phagocyte cytokine synthesis is perhaps their most common moonlighting activity. Friedland and colleagues were the first to show this in 1993 when they reported that the chaperonin 60.2 protein (Cpn60.2, CNP60, $60 \mathrm{kDa}$ chaperonin, Heat Shock Protein 60, Chaperonin 60, HSP-60) of M. tuberculosis stimulated monocytes to secrete pro-inflammatory cytokines [32]. The organisms whose Cpn60 have been studied in detail are Chlamydia pneumoniae, H.pylori, and M. tuberculosis.

II.2.1 Chlamydia pneumonia: C. pneumoniae is a widespread obligate intracellular bacterium with a seropositivity rate of $50 \%$ by 20 years of age increasing to approximately $80 \%$ by 80 years of age [37]. More importantly, C. pneumoniae has been linked to the pathology of atherosclerosis in the capacity of a causative agent [37]. This has drawn attention to the moonlighting actions of chlamydial molecular chaperones. Kol and colleagues [38] first reported on the Cpn60 protein of this bacterium and revealed the presence of C. pneumoniae Cpn60 in atherosclerotic plaques. They also noted the ability of C. pneumoniae Cpn60 to stimulate monocyte pro-inflammatory cytokine synthesis [38]. Subsequently, two important responses were seen after in vivo peritoneal cavity and intra-tracheal administration of chlamydial Cpn60 in a mouse model. The former resulted in increased serum levels of chemokines CXCL1 and CXCL2 and a noticeable build up of neutrophils, while the latter resulted in local accumulation of inflammatory cells and up regulation of cytokine levels [39]. Surprisingly, C. pneumoniae Cpn60 has also been shown to inhibit the generation of gas nitric oxide, a potent bioactive compound, in the human coronary artery vascular endothelial cells. If this were to happen in vivo, it would result in endothelial dysfunction [40].

II.2.2 Helicobacter pylori: The H. pylori chaperonin 60.2 protein has been found to be a major antigen in patients with gastro-duodenal disease with potential diagnostic significance [33]. This immune-genicity is 
thought to be enhanced by the discovery that Cpn60 is a $\mathrm{H}$. pylori cell surface protein [34]. However, of particular interest are the reports that the cell surface H. pylori Cpn60 protein may be involved in the process of gastric carcinoma formation [35] and its function as an adhesion for binding to human epithelial cells [4].

II.2.3 M. tuberculosis: Two Cpn60 proteins (60.1 and 60.2), with 60\% sequence identity, are found in this bacterium [43]. While this generates the hypothesis that these proteins are nearly identical in both structure and function, it is well established that a single amino acid mutation can lead to a marked protein behaviour change. Following on from this, it is not surprising that these two proteins have different biological activities. For example, they bind to different receptors suggesting no competition for binding to the human monocyte cell surface [44]. The potential importance of Cpn60.2 in the infectious process of TB was determined by a series of recent experiments by Hickey and colleagues [45]. They found that Cpn60.2 exists upon the surface of M. tuberculosis cells in significant amounts, as does DnaK, the Hsp70 protein of this organism. To demonstrate whether these proteins played a role in bacterial binding, recombinant versions of Cpn60.2 and DnaK were examined for their ability to inhibit the association of M. tuberculosis bacilli with macrophages. The results showed that recombinant Cpn60.2 can inhibit approximately 57\% of this association with macrophages, while DnaK was not inhibitory at comparable concentrations suggesting that while M. tuberculosis displays both surface-associated Cpn60.2 and DnaK, only Cpn60.2 demonstrates adhesin functionality with regard to macrophage interaction.

In addition, the reported moonlighting activities of the Cpn60.1 protein found in mycobacteria include a role in biofilm formation [46]. Inactivation of the cpn60.1 gene in Mycobacterium smegmatis results in an organism that grows normally in culture but fails to form biofilms at liquid-air interfaces. The same gene inactivation in M. tuberculosis does not result in impairment of biofilm formation [43].

Moreover, the $\mathrm{Hsp} 70$ protein of $\mathrm{M}$. tuberculosis is now gaining evidence indicating that it also has moonlighting properties. CC and CXC chemokines are thought to control the formation of the characteristic granuloma formation found in mycobacterial infections and one component of $\mathrm{M}$. tuberculosis which stimulates chemokine synthesis and activates human myeloid and lymphoid cells is the Hsp70 protein [47-50]. Interestingly, it has been reported that M. tuberculosis DnaK binds to the HIV chemokine receptor type 5 (CCR5) [49] which the virus initially uses to enter and infect host cells. This is an interesting finding, given that there is synergy between infection with HIV and M. tuberculosis [50]. Now, as HIV and M. tuberculosis DnaK (which is present on the bacterial surface) both bind to CCR5, Henderson and Martin [4] pose the question: can DnaK block HIV binding? Surprisingly, the answer is yes, suggesting that this mycobacterial protein may have some therapeutic potential [51].

While it is still early days in the study of bacterial moonlighting proteins, a growing proportion of micro-organisms are being revealed to have multiple genes encoding Cpn60 proteins. Hence, a clear theme is emerging in the role of such proteins. Most of them seem to play some role in bacterial pathogenesis, either by acting as secreted cell signalling agents and/or cell surface bacterial adhesins.

\section{Medical Relevance}

Many authors agree that the participation of moonlighting proteins may be associated to the complex phenotypes of several disorders [11,52]. While concrete evidence is lacking to support such claims, detailed below are two examples of moonlighting proteins that could play a role in human disease.

Mycobacterium tuberculosis glutamate racemase (MurI): Mycobacterium tuberculosis is a highly infectious pathogen responsible for causing tuberculosis in humans [53]. Left untreated, this disease can be fatal. The broad-spectrum $2^{\text {nd }}$ generation fluoroquinolone antibiotic Ciprofloxacin (Alcipro, Ciprobay, Cipro, Ciproxin) can be used to combat M. tuberculosis by stimulating the introduction of double-strand DNA upon binding to a DNA gyrase [54]. However, a moonlighting function of the MurI protein counteracts this effect of ciprofloxacin. Since MurI is a glutamate racemase (essential M. tuberculosis enzyme which is involved in peptidoglycan bio-synthesis), it can catalyse the conversion of L-glutamate to D-glutamate, the peptidoglycan building block [55]. Additionally, MurI can also reduce the binding of gyrase to DNA, essentially functioning as a DNA gyrase inhibitor. This has been demonstrated in numerous bacterial species, including M. tuberculosis [56]. This function is independent of its glutamate racemase enzymatic activity; thus representing a moonlighting property [57]. Because MurI can inhibit the binding of gyrase to DNA (and subsequent introduction of ds-DNA breaks), overproduction of MurI, enzymatically active or not, protects M. tuberculosis against the action of ciprofloxacin [57,11].

Dihydrolipoamide dehydrogenase (DLD): DLD is a mitochondrial enzyme critical for energy metabolism and redox balance that in humans is encoded by the DLD gene. This protein is a subunit of several enzyme complexes (at least five different multi-enzyme complexes). These complexes are essential for the breakdown of certain molecules to produce energy in cells [58]. Severe disorders in infancy, such as pyruvate dehydrogenase deficiency, maple syrup urine disease, hypotonia and metabolic disorders are all associated with deficiency in DLD activity [11]. The severity of symptoms is variable and depends on the mutation present in the DLD gene 
(cytogenetic Location: 7q31-q32). In healthy individuals, the dihydrolipoamide dehydrogenase is primarily present as active homodimers that are in equilibrium with the inactive monomer [59]. However, under certain conditions, such as matrix acidification (mitochondrial), the protein is mainly monomeric, resulting in a decrease in DLD enzyme activity. Babady and colleagues [58] have reported that the destabilisation of the homodimer via mutations may increase the ability of DLD to function as a protease due to enhanced exposure of a catalytic dyad at the dimer interface. This activity is autonomous of its enzymatic function and a single point mutation in the catalytic dyad S456A results in the total loss of proteolytic activity with no affect on enzymatic function. This particular moonlighting role of DLD may contribute in a negative way to the metabolic defects seen in several DLD patients [11].

Lastly, it has also been suggested that moonlighting proteins may have a role in host cell invasion [60]. Due to their possible role in disease, Huberts and van der Klei [11] emphasise that enhancing or blocking moonlighting functions could be a target for future drug design. This advancement would require more in-depth knowledge of the general and molecular functions of moonlighting proteins.

\section{Moonlighting Protiens In Life Sciences}

The awareness of the existence of various moonlighting functions presents a major challenge in genome annotation [11]. In fact, one can assert that even well studied proteins may have additional functions that have yet to be discovered. It is noteworthy to point out that the discovery of numerous moonlighting functions to date has been completely unanticipated $[5,62,61,58$,]. While we can conjure many explanations as to why this may be so, the most predominate and clear-cut notion is simply that moonlighting functions are typically difficult to predict given that many functions do not depend on well known, conserved protein patterns [58]. In actual fact, moonlighting functions are not often conserved [61]. Certainly, there are some examples where specific proteins have differing moonlighting functions in different species: the catalytic enzyme aconitase, found in Saccharomyces cerevisiae, has a second function in mitochondrial DNA maintenance [62], while it has an involvement in iron homeostasis in mammals [63] and M. tuberculosis [64]. Furthermore, aldolase is indispensable in the assembly and activity of the vacuolar H+-ATPase in yeast [65, 66], but its actin binding capabilities are important for host cell invasion in Plasmodium falciparum and Plasmodium vivax [67].

In addition to their unanticipated discoveries, moonlighting proteins have also been discovered using yeast two-hybrid assays [23] and proteomics [4]. Mass spectrometry has also been suggested as a tool for identifying novel moonlighting proteins [68]. Huberts and van der Klei [11] have suggested a new strategy to search directly for moonlighting functions in enzymes. This would involve comparisons between the phenotypes of strains in which the enzyme is inactivated by a point mutation with the strains in which the entire gene is deleted. If the gene encodes a moonlighting protein, one would expect to see a discrepancy between both phenotypes [11].

Another important challenge is the understanding of how moonlighting proteins perform such assorted functions. One strategy reported by a previous review is to identify the residues which are essential for the moonlighting function as in a number of cases; these residues are somewhat easy to find [11]. For example, Yoshida, et al. [61] demonstrated that the toxic Enterobacter aerogenes GroEL and non-toxic Escherichia coli GroEL proteins differ by only 11 amino acids. In this case, it is apparent that some of these 11 residues must be essential for the moonlighting activity. One future aim in the field of moonlighting proteins would be to locate the amino acids crucial for the moonlighting functions in other moonlighting proteins [11].

\section{The Opportunities And Challenges Provided By Moonlighting Proteins For Drug Development}

The participation of moonlighting properties in disease processes offers both therapeutic challenges and opportunities [17]. This effort will require a more in-depth understanding of the mechanistic and structural bases of moonlighting proteins in addition to which functions are significant in which cell types. One main obstacle associated with this 'therapeutic manipulation' of moonlighting proteins is the suppression of the function implicated in pathology while at the same time minimising the disruption of other functions. For example, the activity of the glucose-6-phosphate isomerase (PGI) autocrine motility factor plays multiple roles in malignancy by inhibiting apoptosis, promoting motility and metastasis, and inducing angiogenesis in endothelial cells [68]. Thus, the autocrine motility factor is a good target for chemotherapy, but it is essential that drugs do not interfere with the protein's neuroleukin and maturation factor activities.

In theory, inhibiting specific moonlighting functions such as the cytoplasmic enzymes on the surface of bacteria that are involved in adhesion and immune evasion or cytokine activities of PGI could be easily met as they operate outside the cell. Copley [17] explains that in these cases, due to inhibition of cytoplasmic functions, the use of drugs that cannot get into cells would avoid toxic side effects. This is an fascinating turnaround of the frequent problem of delivering drugs into the cytoplasm of cells. 
A promising therapeutic approach proposed by Yanagawa et al. [68] is targeting interfaces between moonlighting proteins and binding partners involved in pathological processes with small molecules. A handful of drugs targeting specific functions of moonlighting proteins are currently in use or under development. Selegiline (Anipryl, L-deprenyl, Eldepryl, Emsam, Zelapar), is a monoamine oxidase B inhibitor, used to treat early Parkinson's disease. Hara et al. [69] reported that the deprenyl derivative TCH346 shows neuroprotective effects even though it does not inhibit monoamine oxidase, signifying that it has another important target. Deprenyl and TCH346 bind to GAPDH, preventing S-nitrosylation and binding to Siah1. Consequently, the proapoptotic effect, caused by the translocation of GAPDH to the nucleus, is prevented. The net effect is a significant decrease in the level of cell death caused by toxins in both cells and whole animals [69]. Moreover, proteins that moonlight as cytokines, such as the human chaperone Hsp10 which has immunosuppressive effects, could be used as therapeutic agents to modulate the immune system. The efficacy and safety of recombinant $\mathrm{Hsp} 10$ for the treatment of multiple sclerosis [70], rheumatoid arthritis [71], and psoriasis [72] have been evaluated. The literature on the immune-modulatory effects of Hsp10 (and other moonlighting chaperones) is forthcoming which hints that this will be a fruitful field for development of new treatment models for a number of human diseases [73].

\section{Conclusion}

One unavoidable conclusion based upon the many discoveries of moonlighting proteins is that cellular physiology is more complex than previously thought from the viewpoint in which each protein serves a single function. The notion that in order to understand the metabolic and gene regulatory networks, one must understand the molecular functions of the parts, as well as their interactions within the system, underpins the field of systems biology. However, the existence of moonlighting functions complicates this picture because it means that the parts themselves are more complex. Certainly, it can be concluded that protein moonlighting participates in bacterial virulence seen in a variety of pathogens, including M. tuberculosis, C. Pneumonia, H. pylori, and group A streptococci. Likewise, molecular chaperones and main proteins involved in the key metabolic processes also have unanticipated purposes which participate in bacterial virulence. While there is much to learn about the moonlighting proteins and their functions, it is almost certain that more moonlighting functions will be discovered. Much remains to be learned about the molecular details of the processes that regulate the distribution of moonlighting proteins between various locations and functions. Clarification of these details will provide a more realistic picture of how the thousands of proteins in cells interact to provide flexibility to environmental disturbances, and how these interactions go awry in disease. Identifying moonlighting activity is clearly not an easy process, with most of the currently identified bacterial moonlighting proteins being discovered by accident. As more and more data on moonlighting proteins become available, it will become possible to develop statistical, data-mining, and machine-learning approaches to examining these proteins and making predictions. Ultimately, this more complete understanding will allow the development of drugs that are more precisely targeted to specific functions of moonlighting proteins

\section{References}

[1] A Garrod. Inborn Errors of Metabolism. London: Hodder \& Stoughton 1923; Retrieved March 25, 2014, from: http://www.esp.org/books/garrod/inborn-errors/facsimile/

[2] E Tatum, G Beadle. Genetic control of biochemical reactions in Neurospora. Proc Natl Acad Sci USA, 1942(28), 234-243.

[3] C Jeffery. Moonlighting proteins: old proteins learning new tricks. Trends Genet, 19, 2003, 415-7.

[4] B Henderson, A Martin. Bacterial Virulence in the Moonlight: Multitasking Bacterial Moonlighting Proteins Are Virulence Determinants in Infectious Disease. Infection and Immunity, 79(9), 2011, 3476-3491.

[5] J Piatigorsky, W O'Brien, B Norman, K Kalumuck, G Wistow, T Borras, J Nickerson, E Wawrousek. Gene sharing by deltacrystallin and argininosuccinate lyase. Proc Natl Acad Sci USA, 85, 1998, 3479-8343.

[6] J Piatigorsky. Gene sharing, lens crystallins and speculations on an eye/ear evolutionary relationship. Inter Comp Biol, 43, 2003, 492-499.

[7] M Sirover. New insights into an old protein: the functional diversity of mammalian glyceraldehyde-3-phosphate dehydrogenase. Biochimica et Biophysica Acta, 1432, 1992, 159-184.

[8] M Perucho, J Salas, M Salas. Study of the interaction of glyceraldehyde-3-phosphate dehydrogenase with DNA. Biochim Biophys Acta, 606, 1998, 181-195.

[9] D Weaver. Telomeres: moonlighting by DNA repair proteins. Curr Biol, 8, 1998, R492-494.

[10] C Jeffery. Moonlighting proteins. Trends Biochem Sci, 24, 1999, 8-11.

[11] D Huberts, I van der Klei. Moonlighting proteins: An intriguing mode of multitasking. Biochimica et Biophysica Acta, 1803, 2010, 520-525

[12] C Jeffery. Molecular mechanisms for multitasking: recent crystal structures of moonlighting proteins. Curr Opin Struct Biol,14, 2004, 663-668.

[13] C Jeffery. Moonlighting proteins- an update. Mol Biosyst, 5, 2009, 345-350.

[14] J. Piatigorsky, Gene Sharing and Evolution: The Diversity of Protein Functions (Harvard University Press, 2007).

[15] C Gancedo, C Flores. Moonlighting proteins in yeasts. Microbiol Mol Biol Rev, 72, 2008, 197-210.

[16] I Nobeli, A Favia, J Thornton. Protein promiscuity and its implications for biotechnology. Nat Biotechnol, 27, 2009, 157-167.

[17] S Copley. Moonlighting is mainstream: paradigm adjustment required. Bioessays, 34, 2012, 578-588.

[18] S Hernández, I Amela, J Cedano, J Piñol, J Perez-Pons, A Mozo-Villarias, et al. Do Moonlighting Proteins Belong to the Intrinsically Disordered Protein Class? J Proteomics Bioinform, 5(11), 2012, 262-264. 
[19] B Moore. Bifunctional and moonlighting enzymes: lighting the way to regulatory control. Trends Plant Sci, 9, 2004, 221-228.

[20] M Hughes, J Moore, J Santangelo. Identification of major outer surface proteins of Streptococcus agalactiae. Infect Immun, 70, 2002, 1254-1259.

[21] G Boel, H Jin, V Pancholi. Inhibition of cell surface export of group A streptococcal anchorless surface dehydrogenase affects bacterial adherence and ntiphagocytic properties. Infect Immun, 73, 2005, 6237-6248.

[22] V Kainulainen, V Loimaranta, A Pekkala, S Edelman, S Antikainen, R Kylvaja, et al. Glutamine Synthetase and Glucose-6Phosphate Isomerase Are Adhesive Moonlighting Proteins of Lactobacillus crispatus Released by Epithelial Cathelicidin LL-37. Journal of Bact, 195, 2013, 2509-19.

[23] V Pancholi, V Fischetti. A major surface protein on group A streptococci is a glyceraldehyde-3-phosphate-dehydrogenase with multiple binding activity. J Exp Med, 176, 1992, 415-426.

[24] C Jeffery. Mass spectrometry and the search for moonlighting proteins. Mass Spectrom Rev, 24, 2005, 772-782.

[25] E Ling, G Feldman, M Portnoi, R Dagan, K Overweg, F Mulholland, et al. Glycolytic enzymes associated with the cell surface of Streptococcus pneumoniae are antigenic in humans and elicit protective immune responses in the mouse. Clin Exp Immunol, 138, 2005, 290-298.

[26] A Diaz-Ramos, A Roig-Borrellas, A Garcia-Melero, R Lopez-Alemany. $\alpha$-Enolase, a Multifunctional Protein: Its Role on Pathophysiological Situations. Journal of Biomedicine and Biotechnology, 2012, 156795.

[27] V Pancholi, V Fischetti. $\alpha$-Enolase, a novel strong plasmin-(ogen) binding protein on the surface of pathogenic streptococci. J Biol Chem, 273, 1998, 14503-14515.

[28] G Whiting, J Evans, S Patel, S Gillispie. Purification of native $\alpha$-enolase from Streptococcus pneumoniae that binds plasminogen and is immunogenic. J Med Microbiol, 51, 2002, 837-843.

[29] J Sha, T Erova, A Chopra. Surface-expressed enolase contributes to the pathogenesis of clinical isolate SSU of Aeromonas hydrophila. J Bacteriol, 191, 2009, 3095-3107.

[30] M Dinis, D Tavares, I Veiga-Malta, A Fonseca, E Andrade, G Trigo, et al. Oral therapeutic vaccination with Streptococcus sobrinus recombinant enolase confers protection against dental caries in rats. J Infect Dis, 199, 2009, 116-123.

[31] R Spurbeck, C Arvidson. Lactobacillus jensenii surface associated proteins inhibit Neisseria gonorrhoeae adherence to epithelial cells. Infect Immun, 78, 2010, 3103-3111.

[32] J Friedland, R Shattock, D Remick, G Griffin. Mycobacterial 65-kD heat shock protein induces release of proinflammatory cytokines from human monocytic cells. Clin Exp Immunol, 91, 1993, 58-62.

[33] N Yunoki, K Yokota, M Mizuno, Y Kawahara, M Adachi, H Okada, et al. Antibody to heat shock protein can be used for early serological monitoring of Helicobacter pylori eradication treatment. Clin Diagn Lab Immunol, 7, 2000, 574-577.

[34] H Yamaguchi, T Osaki, H Taguchi, T Hanawa, T Yamamoto, S Kamiya. Flow cytometric analysis of the heat shock protein 60 expressed on the cell surface of Helicobacter pylori. J Med Microbiol, 45, 1996, 270-27

[35] L Xue, Q Su, X Mao, Q Lin, X Liu, C Liu C, et al., A Double-Edged Sword: Roles of Helicobacter Pylori in Gastric Carcinoma, In Lotfy M (Ed.), Gastric Carcinoma- Molecular Aspects and Current Advances (Croatia: InTech, 2011) 255-294.

[36] C Atanassov, L Pezennec, A d'Alayer, C Grollier, B Picard, JFauchere. Novel antigens of Helicobacter pylori correspond to ulcerrelated antibody pattern of sera from infected patients. J Clin Microbiol, 40, 2002, 547-552.

[37] C Watson, N Alp. Role of Chlamydia pneumoniae in atherosclerosis. Clin Sci, 114, 2008, 509-531.

[38] A Kol, G Sukhova, A Lichtman, P Libby. Chlamydial heat shock protein 60 localizes in human atheroma and regulates macrophage tumor necrosis factor-alpha and matrix metalloproteinase expression. Circulation, 98, 1998, 300-307.

[39] Y Bulut, K Shimada, M Wong, S Chen, P Gray, R Alsabeh, et al. Chlamydial heat shock protein 60 induces acute pulmonary inflammation in mice via the Toll-like receptor 4- and MyD88- dependent pathway. Infect Immun, 77, 2009, 2683-2690.

[40] C Chen, H Chai, X Wang, P Lin, Q Yao. Chlamydia heat shock protein 60 decreases expression of endothelial nitric oxide synthase in human and porcine coronary artery endothelial cells. Cardiovasc Res, 83, 2009, 768-777.

[41] K Karunakaran, Y Noguchi, T Read, A Cherkasov, J Kwee, C Shen, et al. Molecular analysis of the multiple GroEL proteins of Chlamydiae. J Bacteriol, 185, 2003, 1958-1966.

[42] F Wuppermann, K Molleken, M Julien, C Jantos, J Hegemann. Chlamydia pneumoniae GroEL1 protein is cell surface associated and required for infection of HEp-2 cells. J Bacteriol, 190, 2008, 3757-3767.

[43] Y Hu, B Henderson, P Lund, P Tormay, M Ahmed, S Gurcha, et al. A Mycobacterium tuberculosis mutant lacking the groEL homologue Cpn60.1 is viable but fails to induce an inflammatory response in animal models of infection. Infect Immun, 76, 2008, 1535-1546.

[44] A Cehovin, A Coates, Y Hu, Y Riffo-Vasquez, P Tormay, C Botanch, et al. Comparison of the moonlighting actions of the two highly homologous chaperonin 60 proteins of. Mycobacterium tuberculosis. Infect Immun, 78, 2010, 3196-3206.

[45] T Hickey, L Thorson, D Speert, M Daffe, R Stokes. Mycobacterium tuberculosis Cpn60.2 and DnaK are located on the bacterial surface, where Cpn60.2 facilitates efficient bacterial association with macrophages. Infect Immun, 77, 2009, 3389-3401.

[46] A Ojha, M Anand, A Bhatt, L Kremer, W Jacobs Jnr, J Hatfull. GroEL1: a dedicated chaperone involved in mycolic acid biosynthesis during biofilm formation in mycobacteria. Cell, 123, 2005, 861-873.

[47] T Lehner, L Bergmeier, Y Wang, L Tao, M Sing, R Spallek, et al. Heat shock proteins generate beta-chemokines which function as innate adjuvants enhancing adaptive immunity. Eur J Immunol, 30, 2002, 594-603.

[48] Y Wang, C Kelly, J Karttunen, T Whittall, P Lehner, L Duncan, et al. CD40 is a cellular receptor mediating mycobacterial heat shock protein 70 stimulation of CC-chemokines. Immunity, 15, 2001, 971-983.

[49] R Floto. Dendritic cell stimulation by mycobacterial Hsp70 is mediated through CCR5. Eur Respir Rev, 17(108), 2008, 70-71.

[50] L Bekker, R Wood. The changing natural history of tuberculosis and HIV coinfection in an urban area of hyperendemicity. Clin Infect Dis, 50(3), 2003, S208-S214.

[51] K Babaahmady,W Oehlmann, M Singh, T Lehner. Inhibition of human immunodeficiency virus type 1 infection of human CD4+ T cells by microbial HSP70 and the peptide epitope 407-426. J Virol, 81, 2007, 3354-3360.

[52] G Sriram, J Martinez, E McCabe, J Liao, K Dipple. Single-gene disorders: what role could moonlighting enzymes play? Am. J. Hum. Genet, 76, 2005, 911-924.

[53] B Young, M Perkins, K Duncan, C Barry. Confronting the scientific obstacles to global control of tuberculosis. J Clin Invest, 118, 2008, 1255-1265.

[54] K Drlica, X Zhao. DNA gyrase, topoisomerase IV, and the 4-quinolones. Microbiol Mol Biol Rev, 61, 2007, $377-392$.

[55] T Lundqvist, S Fisher, G Kern, R Folmer, Y Xue, D Newton, et al. Exploitation of structural and regulatory diversity in glutamate racemases. Nature, 447, 2007, 817-822.

[56] S Sengupta, M Shah, V Nagaraja. Glutamate racemase from Mycobacterium tuberculosis inhibits DNA gyrase by affecting its DNA-binding. Nucleic Acids Res, 34, 2006, 5567-5576. 
[57] S Sengupta, S Ghosh, V Nagaraja. Moonlighting function of glutamate racemase from Mycobacterium tuberculosis: racemization and DNA gyrase inhibition are two independent activities of the enzyme. Microbiology, 154, 2008, $2796-2803$.

[58] N Babady, Y Pang, O Elpeleg, G Isaya. Cryptic proteolytic activity of dihydrolipoamide dehydrogenase. Proc Natl Acad. Sci USA, $104,2007,6158-6163$

[59] N Klyachko, V Shchedrina, A Efimov, S Kazakov, I Gazaryan, B Kristal, et al. pH-dependent substrate preference of pig heart lipoamide dehydrogenase varies with oligomeric state: response to mitochondrial matrix acidification. J Biol Chem, 280, 2005, 16106-16114.

[60] M Podobnik, R Tyagi, N Matange, U Dermol, A Gupta, R Mattoo, et al. A mycobacterial cyclic AMP phosphodiesterase that moonlights as a modifier of cell wall permeability. J Biol Chem, 284, 2009, 32846-32857.

[61] N Yoshida, K Oeda, E Watanabe, T Mikami, Y Fukita, K Nishimura, et al. Protein function: Chaperonin turned insect toxin. Nature, 411, 2001, 44

[62] X Chen, X Wang, B Kaufman, R Butow. Aconitase couples metabolic regulation to mitochondrial DNA maintenance. Science, 307, 2005, 714-717.

[63] M Kennedy, L Mende-Mueller, G Blondin, H Beinert. Purification and characterization of cytosolic aconitase from beef liver and its relationship to the iron-responsive element binding protein. Proc Natl Acad Sci USA, 89, 1992, 11730-11734.

[64] S Banerjee, A Nandyala, P Raviprasad, N Ahmed, S Hasnain. Iron-dependent RNA-binding activity of Mycobacterium tuberculosis aconitase, J Bacteriol, 189, 2007, 4046-4052.

[65] M Lu, D Ammar, H Ives, F Albrecht, S Gluck. Physical interaction between aldolase and vacuolar H+-ATPase is essential for the assembly and activity of the proton pump. J Biol Chem, 282, 2007, 24495-24503.

[66] M Lu, L Holliday, L Zhang, W Dunn, S Gluck. Interaction between aldolase and vacuolar H+-ATPase: evidence for direct coupling of glycolysis to the ATP-hydrolyzing proton pump. J Biol Chem, 276, 2001, 30407-30413.

[67] J Bosch, C Buscaglia, B Krumm, B Ingason, R Lucas, C Roach, T Cardozo, V Nussenzweig, W Hol. Aldolase provides an unusual binding site for thrombospondin- related anonymous protein in the invasion machinery of the malaria parasite. Proc Natl Acad Sci USA, 104, 2007, 7015-7020.

[68] T Yanagawa, T Funasaka, S Tsutsumi, H Watanabe, et al. Novel roles of the autocrine motility factor/phosphoglucose isomerase in tumor malignancy. Endocr Relat Cancer, 11, 2004, 749-59.

[69] M Hara, B Thomas, M Cascio, B Bae, L Hester, V Dawson, et al. Neuroprotection by pharmacologic blockade of the GAPDH death cascade. Proc Natl Acad Sci USA, 103, 2006, 3887-9.

[70] S Broadley, D Vanags, B Williams, B Johnson, D Feeney, L Griffiths, et al. Results of a phase IIa clinical trial of an antiinflammatory molecule, chaperonin 10, in multiple sclerosis. Mult Scler, 15, 2009, 329-36.

[71] D Vanags, B Williams, B Johnson, S Hall, P Nash, A Taylor, et al. Therapeutic efficacy and safety of chaperonin 10 in patients with rheumatoid arthritis: a doubleblind randomised trial. Lancet, 368, 2006, 855-63.

[72] B Williams, D Vanags, S Hall, C McCormack, P Foley, J Weiss, et al. Efficacy and safety of chaperonin 10 in patients with moderate to severe plaque psoriasis: evidence of utility beyond a single indication. Arch Dermatol, 144, 2008, 683-5.

[73] B Henderson, A Pockley. Molecular chaperones and proteinfolding catalysts as intercellular signaling regulators in immunity and inflammation. J Leukoc Biol, 88, 2010, 445-62. 